\title{
Violência contra a mulher por parceiro íntimo na pandemia de COVID-19:
}

\section{magnitude e fatores associados}

\author{
Intimate partner violence against women in the COVID-19 pandemic: magnitude and associated \\ factors \\ Violencia de pareja íntima contra mujeres en la pandemia de COVID-19: magnitud y factores \\ asociados
}

Haylane Nunes da Conceição ORCID: https://orcid.org/0000-0001-9508-9615 Centro Universitário de Ciências e Tecnologia do Maranhão, Brasil E-mail: lanenunes_@hotmail.com

Janayra Rodrigues Dantas ORCID: https://orcid.org/0000-0002-1438-1292 Universidade Estadual do Maranhão, Brasil E-mail: janayrarodriguesdantas24@gmail.com

Yuri Guilherme Melo Oliveira ORCID: https://orcid.org/0000-0002-2853-3728 Universidade Estadual do Maranhão, Brasil E-mail: ywrygwilherme18@gmail.com

Guida Graziela Santos Cardoso ORCID: https://orcid.org/0000-0002-8639-7146 Universidade Federal do Piauí, Brasil E-mail: guida.cardoso18@gmail.com

Beatriz Aguiar da Silva

ORCID: https://orcid.org/0000-0001-9904-2080 Universidade Estadual do Maranhão, Brasil E-mail: bia_aguiar12@hotmail.com

Elisá Victória Silva e Silva ORCID: https://orcid.org/0000-0002-7530-1161 Universidade Estadual do Maranhão, Brasil E-mail: elisavitoria1307@gmail.com

Josemeire da Costa Ximenes ORCID: https://orcid.org/0000-0002-6812-4363 Universidade Estadual do Maranhão, Brasil E-mail: jose.meire@hotmail.com

Vitor Emanuel Sousa da Silva ORCID: https://orcid.org/0000-0001-7002-3572 Universidade Estadual do Maranhão, Brasil E-mail: vemanue10612@gmail.com

Rosângela Nunes Almeida

ORCID: https://orcid.org/0000-0001-5152-2800

Universidade Estadual do Maranhão, Brasil E-mail: rnadasilva@hotmail.com

Daniela Reis Joaquim de Freitas ORCID: https://orcid.org/0000-0002-5632-0332 Universidade Federal do Piauí, Brasil E-mail: danielarjfreitas@ufpi.edu.br

Hayla Nunes da Conceição ORCID: https://orcid.org/0000-0001-6035-8280

Universidade Estadual do Maranhão, Brasil E-mail: haylanunes_cx@hotmail.com.

\section{Resumo}

Objetivo: Abordar a magnitude e os fatores associados da violência por parceiro íntimo contra a mulher na pandemia da COVID-19. Metodologia: Trata-se de uma revisão integrativa de literatura, realizada por meio da busca na base de dados MEDLINE, utilizando os descritores "violence", "woman" e "COVID-19", associados ao operador booleano $A N D$. Foram incluídos estudos disponíveis na íntegra, publicado no recorte temporal compreendendo o período de março/2020 e abril/2021 e nos idiomas português, inglês e espanhol, resultando em uma amostra de 10 artigos. 
Resultados: A partir da amostra selecionada, pôde-se constatar que a pandemia de COVID-19 aumentou a frequência e a gravidade dos atos violentos cometidos pelo parceiro íntimo contra a mulher, sendo que a violência psicológica, física e sexual foram os tipos mais relatados por elas. Além disso, o período pandêmico desencadeou o surgimento de novas formas de abusos. O aumento do tempo com o abusador no domicílio, fatores econômicos e a interrupção ou dificuldade de acesso aos serviços de apoio foram apontados como os principais responsáveis pela ocorrência e/ou agravamento da violência perpetrada pelo parceiro íntimo contra mulher durante a pandemia de COVID-19. Conclusão: As informações referentes a magnitude e os fatores associados à violência por parceiro íntimo contra a mulher durante a pandemia poderão auxiliar no desenvolvimento de estratégias preventivas/combate a ocorrência deste tipo de violência, além de colaborar para o planejamento de ações que assegurem a assistência das mulheres em outras situações de emergência semelhante.

Palavras-chave: Violência por parceiro íntimo; Violência contra a mulher; COVID-19.

\begin{abstract}
Objective: To address the magnitude and associated factors of intimate partner violence against women in the COVID-19 pandemic. Methodology: This is an integrative literature review, performed by searching the MEDLINE database, using the descriptors "violence", "woman" and "COVID-19", associated with the Boolean operator AND. Studies available in full were included, published in the time frame covering the period of March/2020 and April/2021 and in Portuguese, English and Spanish, resulting in a sample of 10 articles. Results: From the selected sample, it could be seen that the COVID-19 pandemic increased the frequency and severity of violent acts committed by the intimate partner against women, with psychological, physical and sexual violence being the most reported types For them. Furthermore, the pandemic period triggered the emergence of new forms of abuse. The increase in time with the abuser at home, economic factors and the interruption or difficulty in accessing support services were identified as the main factors responsible for the occurrence and/or worsening of violence perpetrated by an intimate partner against women during the COVID-19 pandemic. Conclusion: Information regarding the magnitude and factors associated with intimate partner violence against women during the pandemic may help to develop strategies to prevent / combat the occurrence of this type of violence, in addition to contributing to the planning of actions to ensure assistance of women in other similar emergency situations.
\end{abstract}

Keywords: Intimate partner violence; Violence against women; COVID-19.

\title{
Resumen
}

Objetivo: Abordar la magnitud y los factores asociados de la violencia infligida por la pareja íntima contra las mujeres en la pandemia de COVID-19. Metodología: Se trata de una revisión integradora de la literatura, realizada mediante la búsqueda en la base de datos MEDLINE, utilizando los descriptores "violencia", "mujer" y "COVID-19", asociados al operador booleano AND. Se incluyeron los estudios disponibles íntegramente, publicados en el período de marzo / 2020 y abril / 2021 y en portugués, inglés y español, resultando una muestra de 10 artículos. Resultados: De la muestra seleccionada se pudo apreciar que la pandemia COVID-19 incrementó la frecuencia y severidad de los actos violentos cometidos por la pareja íntima contra la mujer, siendo la violencia psicológica, física y sexual los tipos más denunciados para ellas. Además, el período de la pandemia provocó la aparición de nuevas formas de abuso. El aumento del tiempo con el abusador en el hogar, los factores económicos y la interrupción o dificultad en el acceso a los servicios de apoyo fueron identificados como los principales factores responsables de la ocurrencia y / o agravamiento de la violencia perpetrada por un compañero íntimo contra la mujer durante la pandemia COVID-19. . Conclusión: La información sobre la magnitud y los factores asociados a la violencia de la pareja íntima contra la mujer durante la pandemia puede ayudar a desarrollar estrategias para prevenir / combatir la ocurrencia de este tipo de violencia, además de contribuir a la planificación de acciones para asegurar la atención de las mujeres en otras situaciones de emergencia similares.

Palabras clave: Violencia de pareja; Violencia contra la mujer; COVID-19.

\section{Introdução}

A violência por parceiro íntimo (IPV - intimate partner violence) contra a mulher corresponde aos atos violentos, de natureza física, sexual e/ ou psicológica perpetrados pelo companheiro atual ou anterior (Paulson, 2020). Em todo o mundo, uma em cada três mulheres já vivenciaram situações de abusos físicos, sexuais ou ambos provocados pelo companheiro (Gebrewahd, Gebremeskel, \& Tadesse, 2020). Este tipo de violência pode desencadear diversos desfechos negativos para a saúde da mulher, entre eles a depressão, uso abusivo de álcool e drogas, infecções sexualmente transmissíveis, maiores risco de lesões físicas, isolamento e limitações no autocuidado (GIBBS et al., 2018; Medonça \& Ludemir, 2017).

Muitos fatores podem ser elencados como fatores de risco para VIP contra mulheres, tais como: baixo nível de escolaridade, juventude, ser separada ou divorciada do agressor (Rosa, Ramos, Gomes, Melo, \& Melo, 2018) e a ocorrência de 
situações de emergência como epidemias (Fawole, Okedare, \& Reed, 2021). As epidemias podem impactar a vida social e econômica das pessoas, favorecendo o aumento da violência contra as mulheres, como ocorreu durante as epidemias do vírus Ebola e Zika (Agüero, 2020; Fawole, Okedare, \& Reed, 2021).

Similarmente ao que houve em epidemias anteriores, a pandemia de COVID-19, ocasionada pelo vírus SARS-CoV-2, também acarretou um notável aumento dos casos de IPV contra mulher em todo o mundo (Fawole et al., 2021; Gebrewahd, Gebremeskel, \& Tadesse, 2020). Diante deste cenário, compreender a magnitude e os fatores associados da IPV contra a mulher durante a pandemia da COVID-19 poderão auxiliar no planejamento de estratégias que previnam/combatam a ocorrência dos atos violentos no momento pandêmico, além de colaborar para o planejamento de ações que assegurem a assistência às mulheres vítimas de VIP em outras situações de emergência semelhante. Desse modo, o presente estudo teve como objetivo abordar a magnitude e os fatores associados da violência por parceiro intimo contra a mulher durante a pandemia de COVID-19.

\section{Metodologia}

Trata-se de uma revisão integrativa da literatura, do tipo exploratória e descritiva. O percurso metodológico foi estruturado em seis etapas recorrentes e interdependentes, sendo elas: elaboração da questão norteadora; busca na literatura; categorização dos estudos; avaliação dos estudos incluídos na revisão integrativa e interpretação dos resultados e síntese do conhecimento (Souza, Silva, \& Carvalho, 2010).

A elaboração da questão norteadora de pesquisa foi fundamentada no acrônimo PICo (participantes, fenômeno de interesse e contexto do estudo) (Santos, Pimenta, \& Nobre, 2007), onde conferiu-se a "P"- Mulheres; "I"- Violência contra a mulher; "Co"- pandemia da COVID-19, originando a seguinte questão norteadora: Qual a magnitude e os fatores associados da violência por parceiro íntimo contra a mulher na pandemia de COVID-19?

Para a identificação dos estudos acerca da referida problemática foi realizado um levantamento por dois pesquisadores, de forma independente, no mês de abril de 2021, na base de dados Medical Literature Analysis and Retrieval System Online (MEDLINE) via US National Library of Medicine (PUBMED). As buscas foram direcionadas por meio da utilização de descritores padronizados e indexados nos Medical Subject Headings (MeSH): "violence", "woman" e "COVID19". Para restringir a amostra juntamente com os termos selecionados e padronizados pelo MeSH foi utilizado o operador booleano "AND". Foram incluídos os estudos disponíveis na íntegra; publicado no recorte temporal compreendendo o período de março/2020 e abril/2021 e nos idiomas português, inglês e espanhol. Foram excluídos os artigos de revisão, protocolos e recomendações, resumos, textos incompletos e os artigos que não abordavam a temática proposta.

Após a seleção, os artigos elegíveis para este estudo foram categorizados por meio da matriz de síntese, elaborada no software Microsoft Excel®. A matriz de síntese foi feita de forma descritiva, englobando os seguintes itens: autor e ano; tipo de estudo/ nível de evidência; local do estudo; perfil amostral e principais resultados. O nível de evidência permitiu classificar os artigos de acordo com seis níveis: (I) revisão sistemática ou metanálise; (II) ensaios clínicos randomizados; (III) ensaios clínicos sem randomização; (IV) estudos de coorte e de caso-controle; (V) revisão sistemática de estudos descritivos e qualitativos; (VI) único estudo descritivo ou qualitativo e (VII) opinião ou consenso (Fineout-Overholt, Melnyk, Stillwell, \& Williamson, 2010). Para facilitar a identificação, os artigos foram codificados, sucessivamente, de E1 a E10.

Por fim, as informações foram analisadas criticamente pelos pesquisadores e comparadas com bibliografias atualizadas sobre a temática. 


\section{Resultados}

Foram identificados, mediante ao cruzamento dos descritores na base de dados, 154 artigos. Destes, após a leitura de títulos e resumos fundamentada nos critérios de elegibilidade, foram excluídos 137, resultando em uma amostra de 17 artigos para a leitura na íntegra. Após a leitura completa, foram selecionados 10 estudos para integrarem esta revisão de literatura (Figura 1).

Figura 1: Fluxograma das etapas de busca e seleção dos artigos analisados. Caxias/MA, Brasil, 2021.

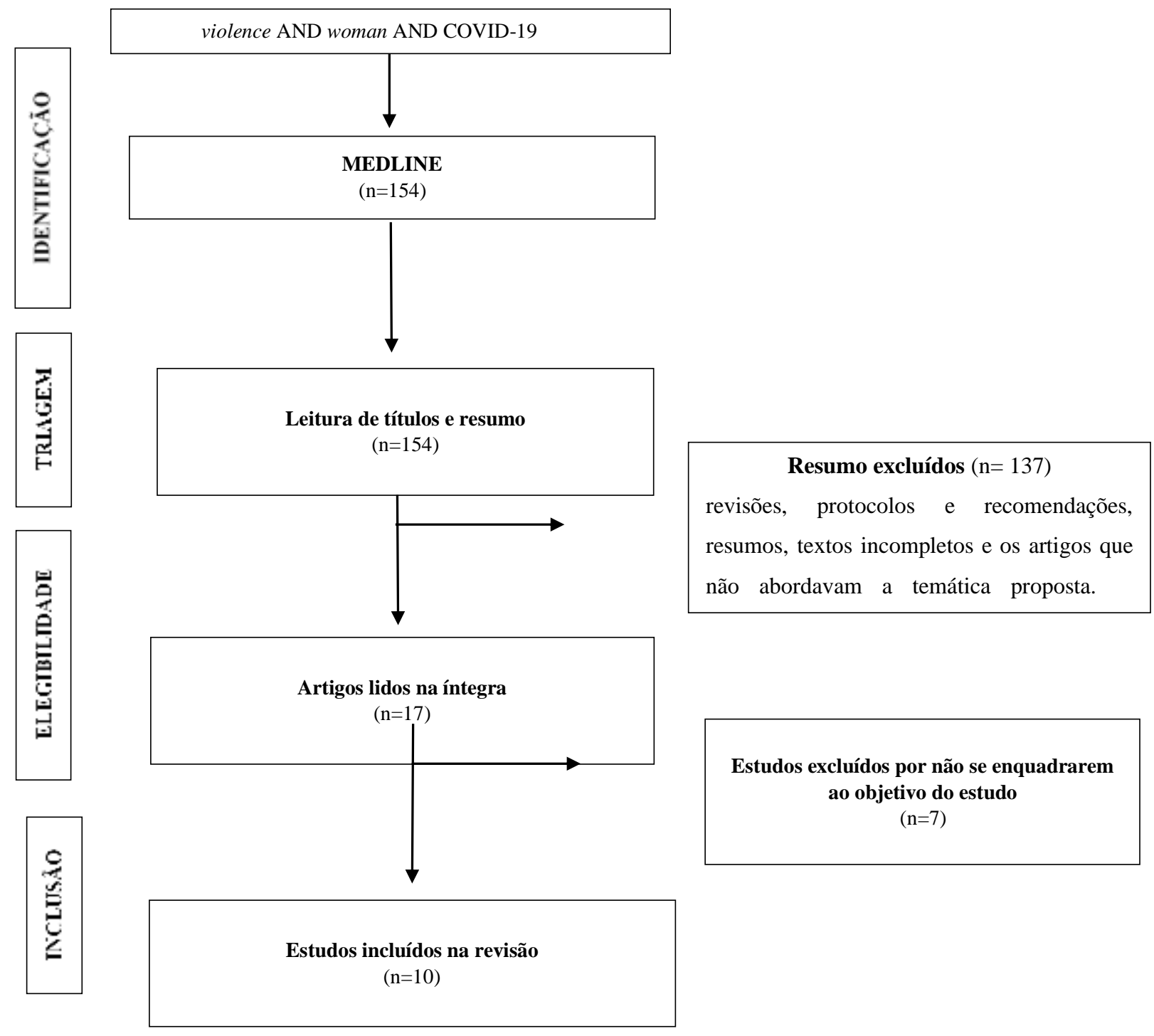

Fonte: Autores.

\section{Características dos artigos analisados}

Em relação ao ano de publicação, observou-se que a maioria dos artigos incluídos foram publicados em 2021 (n=7; 70\%). Quanto ao local do estudo, verificou-se uma predominância de estudos feitos nos Estados Unidos da América (EUA), com três $(30 \%)$ publicações. As demais pesquisas foram desenvolvidas na Nigéria, Iraque, Etiópia, Jordânia, Irã, Paquistão e Índia, sendo constando um (10\%) artigo para cada um desses países. Quanto ao delineamento da pesquisa, houve uma maior prevalência de estudos transversais $(n=7 ; 70 \%)$, seguido por estudo longitudinal retrospectivo, estudo qualitativo e relato de 
caso, com um artigo (10\%) cada. A avaliação do nível de evidência revelou que a grande maioria dos estudos (n=9; 90\%) são classificados no nível VI de evidência científica (Quadro 1).

Com relação a ocorrência de IPV durante a pandemia de COVID-19, observou-se que todos os estudos evidenciaram situações abusivas perpetradas pelo parceiro íntimo (Aolymat, 2021; Fawole et al., 2021; Gebrewahd et al., 2020; Gosangi et al., 2021; Haq, Raza \& Mahmood, 2020; Krishnamurti et al., 2021; Mahmood et al., 2021; Naghizadeh, Mirghafourvand, Mohammadirad, 2021; Pattojoshi et al., 2021; Sabri et al., 2020). As taxas de IPV durante o período pandêmico variaram entre 18,1\% na Índia (Pattojoshi et al., 2021) até 38,7\% no Iraque (Mahmood et al., 2021). No Irã (Naghizadeh et al., 2021) e no Paquistão (Haq et al., 2020) as pesquisas demonstraram uma prevalência de aproximadamente 35\%, enquanto na Etiópia os casos de violência por parceiro íntimo representaram 24,6\% (Gebrewahd et al., 2020) (Quadro 1).

Seis (60\%) artigos revelaram que a pandemia proporcionou um aumento da frequência de IPV em mulheres que já eram vítimas desse tipo de violência antes do período pandêmico (Aolymat, 2021; Gosangi et al., 2021; Krishnamurti et al., 2021; Mahmood et al., 2020; Pattojoshi et al., 2021; Sabri et al., 2020). Um estudo realizado na Jordânia identificou que em uma amostra de 200 mulheres, 20,5\% delas vivenciaram um aumento das práticas abusivas desde o início da pandemia (Aolymat, 2021). Maior prevalência foi encontrada na Índia, onde o aumento da violência foi relatado por 77,6\% das mulheres com histórico de VIP (Pattojoshi et al., 2021). Nos EUA a ocorrência de IPV física foi 1,8 vezes maior em $2020 \mathrm{em}$ comparação com os três anteriores (Gosangi et al., 2021). No Iraque, a prevalência de IPV aumentou 6,6\%, passando de 32,1 para 38,7\% (Mahmood et al., 2020) (Quadro 1).

Três (30\%) estudos demonstraram que a gravidade dos atos violentos contra a mulher foi acentuada com a pandemia (Fawole et al., 2021; Gosangi et al., 2021; Sabri et al., 2020). O aumento da gravidade dos abusos foi identificado na Nigéria (Fawole et al., 2021) e nos EUA (Gosangi et al., 2021; Sabri et al., 2020)(Quadro 1).

Em relação aos tipos de IPV, identificou-se a ocorrência de violência psicológica $(n=7 ; 70 \%)$, violência física $(n=7$; $70 \%)$, violência sexual $(\mathrm{n}=6 ; 60 \%)$ e violência econômica $(\mathrm{n}=1 ; 10 \%)$. Além desses tipos de IPV, um (10\%) estudo constatou a presença de novas formas de violência, estando estas relacionadas a ações que utilizaram o bloqueio decorrente da pandemia de COVID-19 para ameaçar a segurança e a capacidade das mulheres de se protegerem do vírus (Quadro 1).

A violência psicológica, em cinco (50\%) dos artigos incluídos, foi o tipo de IPV mais frequente A prevalência de IPV psicológica variou de 13,3\% na Etiópia (Gebrewahd et al., 2020) a 35\% no Iraque (Mahmood et al., 2020). Em três (30\%) dos estudos, a IPV física e sexual foram, respectivamente, o segundo e terceiro tipo de violência mais comumente relatadas pelas mulheres (Gebrewahd et al., 2020; Mahmood et al., 2020; Pattojoshi et al., 2021) (Quadro 1).

No que se refere aos fatores associados a IPV durante a pandemia, observou-se que a presença de parceiros abusivos em casa ( $\mathrm{n}=2 ; 20 \%$ ) (Aolymat et al., 2021; Sabri et al., 2020), fatores econômicos como desemprego e diminuição da renda do parceiro ( $\mathrm{n}=5$; 50\%) (Aolymat et al., 2021; Fawole et al., 2021, Naghizadeh et al., 2021; Pattojoshi et al., 2021; Sabri et al., 2020) e a interrupção ou dificuldade de acesso aos serviços de apoio ( $\mathrm{n}=3$; 30\%) (Aolymat et al., 2021; Fawole et al., 2021; Sabri et al., 2020) foram os principais responsáveis pela ocorrência e/ou agravamento da IPV contra mulher durante a pandemia de COVID-19 (Quadro 1). 
Quadro 1: Sinopse dos dados dos estudos incluídos na revisão de literatura. Caxias/MA, Brasil, 2021.

\begin{tabular}{|c|c|c|c|c|c|}
\hline Estudo & Autor e ano & $\begin{array}{l}\text { Tipo de estudo/ } \\
\text { Nível de evidência }\end{array}$ & Local do estudo & Perfil amostral & Principais resultados \\
\hline E1 & $\begin{array}{l}\text { Fawole;Okedar } \\
\text {; Reed, } 2021\end{array}$ & Relato de caso/ IV & Nigéria & $\begin{array}{l}\text { Mulheres vítimas de } \\
\text { VIP durante o } \\
\text { bloqueio da COVID- } \\
19(n=7)\end{array}$ & $\begin{array}{l}\text { - Identificou-se que a violência } \\
\text { psicológica, física, econômica e } \\
\text { sexual praticadas pelo companheiro } \\
\text { antes do confinamento aumentaram } \\
\text { em gravidade com a pandemia. Além } \\
\text { disso, houve a ocorrência de novos } \\
\text { tipos de violência. } \\
\text { - O isolamento social, a falta de } \\
\text { acesso aos serviços de apoio e os } \\
\text { estressores econômicos foram fatores } \\
\text { agravantes ou desencadeantes da VIP } \\
\text { relatados pelas vítimas. }\end{array}$ \\
\hline E2 & $\begin{array}{l}\text { Mahmood et } \\
\text { al., } 2021\end{array}$ & $\begin{array}{c}\text { Estudo transversal/ } \\
\text { VI }\end{array}$ & Curdistão, Iraque & $\begin{array}{l}\text { Mulheres casadas } \\
\text { com } 18 \text { anos ou mais } \\
(n=346)\end{array}$ & $\begin{array}{l}\text { - A violência contra a mulher } \\
\text { aumentou de } 32,1 \% \text { para } 38,7 \% \\
\text { durante o bloqueio. } \\
\text { - As formas de violência mais } \\
\text { frequentemente relatadas foram } \\
\text { violência psicológica }(35 \%) \text {, física } \\
(17,6 \%) \text { e sexual }(9,5 \%) \text {, } \\
\text { respectivamente. }\end{array}$ \\
\hline E3 & $\begin{array}{l}\text { Krishnamurt et } \\
\text { al., } 2021\end{array}$ & $\begin{array}{c}\text { Estudo transversal/ } \\
\text { VI }\end{array}$ & EUA & $\begin{array}{lr}\begin{array}{l}\text { Mulheres } \\
\text { residentes }\end{array} \\
\text { Alleghenvy, } \\
\begin{array}{l}\text { Pensilvânia } \\
\text { utilizaram }\end{array} \\
\text { aplicativo } \\
\begin{array}{l}\text { MyHealthyPregnanc } \\
\text { y(n=552) }\end{array}\end{array}$ & $\begin{array}{l}\text { Houve um pequeno aumento na } \\
\text { incidência de violência psicológica, } \\
\text { física e sexual durante a ordem de } \\
\text { acolhimento no local. }\end{array}$ \\
\hline E4 & $\begin{array}{l}\text { Gebrewahd; } \\
\text { Gebremeskel; } \\
\text { Tadesse, } 2020\end{array}$ & $\begin{array}{c}\text { Estudo transversal/ } \\
\text { VI }\end{array}$ & Aksum, Etiópia & $\begin{array}{l}\text { Mulheres em idade } \\
\text { reprodutiva } \\
(\mathrm{n}=682)\end{array}$ & $\begin{array}{l}\text { - } 24,6 \% \text { das participantes relataram } \\
\text { ter sofrido todos as formas de VIP } \\
\text { durante a pandemia. } \\
\text { - A violência psicológica foi o tipo } \\
\text { mais prevalente, com } 13,3 \% \text {, seguido } \\
\text { pela violência física }(8,3 \%) \text { e } \\
\text { violência sexual }(5,3 \%) \text {. }\end{array}$ \\
\hline E5 & $\begin{array}{l}\text { Sabri et al., } \\
\quad 2020\end{array}$ & $\begin{array}{c}\text { Estudo qualitativo/ } \\
\text { VI }\end{array}$ & EUA & $\begin{array}{l}\text { Mulheres imigrantes, } \\
\text { com histórico de } \\
\text { violência por } \\
\text { parceiro íntimo } \\
(\mathrm{n}=45) \text { e prestadores } \\
\text { de serviço } \\
(\mathrm{n}=17)\end{array}$ & $\begin{array}{l}\text { - A pandemia aumentou a frequência } \\
\text { e a gravidade da VIP nas mulheres em } \\
\text { relacionamentos abusivos. } \\
\text { - Desemprego, atrasos na imigração, } \\
\text { interrupção dos serviços de apoio } \\
\text { presenciais e a presença de parceiros } \\
\text { abusivos em casa durante o } \\
\text { isolamento social contribuiu para } \\
\text { aumentar a ocorrência da violência. }\end{array}$ \\
\hline E6 & Aolymat, 2021 & $\begin{array}{c}\text { Estudo transversal/ } \\
\text { VI }\end{array}$ & Jordânia & $\begin{array}{l}\text { Mulheres casadas, } \\
\text { maiores de } 18 \text { anos } \\
(\mathrm{n}=200)\end{array}$ & $\begin{array}{l}\text { - O aumento da violência doméstica } \\
\text { durante a pandemia foi relatado por } \\
20,5 \% \text { das mulheres. } \\
\text { - A perda do emprego ou redução da } \\
\text { renda, maior tempo com os parceiros } \\
\text { em casa e a interrupção dos serviços } \\
\text { de saúde foram fatores que } \\
\text { contribuíram para o agravamento da } \\
\text { VIP durante a pandemia. }\end{array}$ \\
\hline
\end{tabular}




\begin{tabular}{|c|c|c|c|c|c|}
\hline E7 & $\begin{array}{c}\text { Naghizade; } \\
\text { Mirghafourvan } \\
\text {; } \\
\text { Mohammadira } \\
\text { d, } \\
2021\end{array}$ & $\begin{array}{c}\text { Estudo transversal/ } \\
\text { VI }\end{array}$ & Tabriz, Irã & $\begin{array}{ll}\text { Gestantes } & \text { do } \\
\text { ambulatório } & \text { de } \\
\text { obstetrícia } & \text { do } \\
\text { Hospital } & 29-B a h m a n \\
(n=250) & \end{array}$ & $\begin{array}{l}\text { - Os resultados do estudo } \\
\text { demostraram que } 35,2 \% \text { das gestantes } \\
\text { foram expostas a violência doméstica } \\
\text { durante a pandemia. } \\
\text { - Os tipos de violência mais relatadas } \\
\text { foram a violência psicológica } \\
(32,8 \%) \text {, seguida pela violência } \\
\text { sexual }(12,4 \%) \text { e física }(4,8 \%) \text {. } \\
\text { - A violência doméstica esteve } \\
\text { relacionada a diminuição da renda do } \\
\text { parceiro. }\end{array}$ \\
\hline E8 & $\begin{array}{l}\text { Haq; Raza; } \\
\text { Mahmood, } \\
2020\end{array}$ & $\begin{array}{c}\text { Estudo transversal/ } \\
\text { VI }\end{array}$ & Paquistão & $\begin{array}{l}\text { Mulheres casadas } \\
(\mathrm{n}=389)\end{array}$ & $\begin{array}{l}\text { - } 35 \% \text { das mulheres relataram ter } \\
\text { sofrido alguma forma de violência } \\
\text { durante a pandemia. } \\
\text { - A violência psicológica foi o tipo } \\
\text { mais relatado, com } 17 \% \text {. }\end{array}$ \\
\hline E9 & $\begin{array}{c}\text { Gosangi et al., } \\
2021\end{array}$ & $\begin{array}{l}\text { Estudo longitudinal } \\
\text { retrospectivo/ VI }\end{array}$ & EUA & $\begin{array}{l}\text { Vítimas de VIP física } \\
\text { atendidas pelo } \\
\text { programa } \\
\text { institucional } \\
\text { intervenção } \\
\text { prevenção e entra } \\
\text { violência, em um } \\
\text { centro médico, } \\
\text { durante os anos de } \\
2017,2018,2019 \text { e } \\
2020(\mathrm{n}=68) \text {. }\end{array}$ & $\begin{array}{l}\text { - A incidência de VPI física foi } 1,8 \\
\text { vezes maior em } 2020 \text { quando } \\
\text { comparada com os anos anteriores. } \\
\text { - A gravidade das lesões foi maior } \\
\text { durante a pandemia, havendo } 10 \\
\text { vítimas com lesões graves e } 28 \\
\text { profundas em } 2020 \text { contra sete lesões } \\
\text { graves e } 16 \text { profundas de } 2017 \text { a } \\
2019 \text {. }\end{array}$ \\
\hline E10 & $\begin{array}{c}\text { Pattojoshi } \text { et } \\
\text { al., } 2021\end{array}$ & $\begin{array}{c}\text { Estudo transversal/ } \\
\text { VI }\end{array}$ & Índia & $\begin{array}{l}\text { Mulheres casadas } \\
\text { que responderam à } \\
\text { pesquisa online } \\
(\mathrm{n}=560) .\end{array}$ & $\begin{array}{l}\text { - A taxa de VIP durante a pandemia } \\
\text { foi de } 18,1 \% \text {. } \\
\text { - A violência psicológica, física e } \\
\text { sexual foram, respectivamente, as } \\
\text { mais comuns durante o bloqueio. } \\
\text { - Das } 76 \text { mulheres que vivenciavam } \\
\text { violência conjugal antes do bloqueio, } \\
77,6 \% \text { (n= 59) relataram um aumento } \\
\text { na violência desde que o bloqueio foi } \\
\text { aplicado. } \\
\text { - Interrupção de empregos e meios de } \\
\text { subsistência e abstinência forçada de } \\
\text { substancias foram as principais } \\
\text { justificativas das vítimas para o } \\
\text { aumento da violência. }\end{array}$ \\
\hline
\end{tabular}

Fonte: Autores.

\section{Discussão}

A IPV contra a mulher constitui um importante problema social e de saúde pública em todo o mundo (Mascarenhas et al., 2020). Os achados deste estudo identificaram que com a pandemia da COVID-19 a magnitude desta violência, em frequência e gravidade, foi exacerbada em vários países. Estes resultados, embora alarmantes, não são inesperados. A literatura evidencia que o aumento das taxas de IPV está diretamente relacionado a ocorrência de desastres naturais ou outros eventos extremos (Vora, Malathesh, Das, \& Chatterjee, 2020). Esse fato foi observado, por exemplo, após o tsunami do Oceano Índico em 2004, que proporcionou um aumento de 48\% dos casos de IPV entre os anos de 2005 a 2015 na Índia (Krishnakumar \& Verma, 2021). Descobertas semelhantes foram encontradas depois do furacão Katrina nos EUA, onde o número de casos de IPV contra a mulher, após nove meses do furacão, foi triplicado (Silva et al., 2020). 
Durante a pandemia da COVID-19, constatou-se que a violência psicológica foi a mais comumente relatada (Gebrewahd et al., 2020; Haq et al., 2020; Mahmood et al., 2021; Naghizadeh et al., 2021; Pattojoshi et al., 2021), seguida pela violência física, sexual(Gebrewahd et al., 2020; Mahmood et al., 2021; Pattojoshi et al., 2021), econômica e novos tipos de violência (Fawole et al., 2021). A elevada prevalência dos abusos psicológicos entre todas as formas de IPV contra a mulher no período pandêmico é semelhante ao encontrado em pesquisas realizadas nos anos anteriores a pandemia. No Irã, entre os anos de 2013 a 2014, a IPV psicológica foi a forma mais prevalente com 44,4\% (Jahromi, Jamali, Koshkaki, \& Javadpour, 2015), enquanto que no município de Recife, Pernambuco, Brasil, no mesmo período, o abuso psicológico contra a mulher foi o tipo de IPV com maior frequência, correspondendo a 11,7\% (Silva, Lima, \& Ludermir, 2017).

No presente estudo, observou-se ainda o surgimento de novas formas de IPV contra a mulher durante a pandemia de COVID-19. Os novos tipos de violência estiveram relacionados a ações que utilizaram o bloqueio para ameaçar a segurança e a capacidade das mulheres de se protegerem do vírus. Os comportamentos violentos do agressor incluíram impedir a mulher de entrar na casa durante a ordem noturna de recolhimento obrigatório e intimidar as vítimas com ameaças de expulsão da residência durante o bloqueio (Fawole et al., 2021).

Com relação aos fatores associados ao aumento de IPV durante a pandemia, constatou-se que os principais responsáveis foram a presença de parceiros abusivos em casa (Aolymat, 2021; Sabri et al., 2020), fatores econômicos (Aolymat, 2021; Fawole et al., 2021; Naghizadeh et al., 2020; Sabri et al., 2020) e a interrupção ou dificuldade de acesso aos serviços de apoio (Aolymat, 2021; Fawole et al., 2021; Sabri et al., 2020). Com a instauração das medidas de isolamento social, as mulheres passaram a conviver por mais tempo com seu abusador no domicílio(Aolymat, 2021; Sabri et al., 2020. O domicílio é frequentemente relatado como principal local de ocorrência das mais variadas formas de IPV contra a mulher (Mascarenhas et al., 2020). Desse modo, para mulheres que já estão em relacionamentos abusivos, ou em risco sofrerem abuso, as ordens de permanecia em casa aumentaram o risco de violência praticada pelo parceiro íntimo (Roesch et al., 2020).

Outro fator apontado como agravante/desencadeante da IPV durante a pandemia de COVID-19 foram os fatores econômicos relacionados ao desemprego e diminuição da renda (Aolymat, 2021; Fawole et al., 2021; Naghizadeh et al., 2020; Pattojoshi et al., 2021; Sabri et al., 2020). Na carência de recursos financeiros, há uma limitação do acesso aos itens de necessidade básica como alimentação e produtos de higiene, ocasionando um estresse adicional na relação com o parceiro e, consequentemente, favorecendo a ocorrência de episódios de violência do homem contra a companheira (Roesch et al., 2020; Silva et al., 2020).

Um outro fator atribuído a ocorrência de IPV no período pandêmico foi a interrupção ou dificuldade de acesso aos serviços de apoio (Aolymat, 2021; Fawole et al., 2021; Sabri et al., 2020). O rompimento das relações abusivas e a busca por ajuda diante dos atos violentos perpetrados pelo parceiro íntimo no contexto da pandemia de COVID-19 foram dificultados pelo distanciamento das relações sociais com familiares, vizinhos e amigos, pela suspensão ou redução das atividades em igrejas, creches, escolas e serviços de apoio, bem como pela priorização dos serviços de saúde em prestar assistência as pessoas acometidas pelo vírus SARS-CoV-2 (Marques, Moraes, Hasselmann, Deslandes, \& Reichenheim, 2020).

Ressalta-se que o presente estudo apresenta limitações que devem ser consideradas. Primeiro, a seleção dos artigos foi realizada em apenas uma base de dados, o que pode ter diminuído a amplitude de análise. Segundo, mesmo sendo a VPI uma temática extremamente relevante, a investigação foi conduzida no contexto da pandemia da COVID-19, que é relativamente recente, e em decorrência disso poucos estudos primários foram encontrados.

\section{Considerações Finais}

A pandemia da COVID-19 aumentou a frequência e a gravidade dos atos violentos cometidos pelo parceiro íntimo contra a mulher, sendo que a violência psicológica, física e sexual foram os tipos de IPV mais relatadas pelas vítimas. Além 
disso, o período pandêmico desencadeou o surgimento de novas formas de abusos. O aumento do tempo com o abusador no domicílio, fatores econômicos e a interrupção ou dificuldade de acesso aos serviços de apoio foram apontados como os principais responsáveis pela ocorrência e/ou agravamento da IPV contra mulher durante a pandemia.

Demonstrar a magnitude e os fatores associados da IPV contra a mulher durante a pandemia de COVID-19 poderá promover a conscientização e sensibilização na comunidade, pesquisadores, organizações não governamentais, Estado e demais autoridades competentes sobre o quadro alarmante de taxas crescentes de IPV durante o momento pandêmico vivenciado, auxiliando no desenvolvimento de estratégias preventivas e de combate que respondam a ocorrência deste agravo na pandemia. Dentre as estratégias que podem ser promovidas, destaca-se a divulgação de informações sobre a temática nos meios de comunicação e a instauração de medidas de combate a IPV nos planos de resposta a COVID-19.

\section{Referências}

Agüero, J. M. (2020). COVID-19 and The Rise of Intimate Partner Violence. World Development, 137. 10.1016/ j.worlddev.2020.105217.

Aolymat, I. A. (2021). Cross-Sectional Study of the Impact of COVID-19 on Domestic Violence, Menstruation, Genital Tract Health, and Contraception Use among Women in Jordan. American Journal of Tropical Medicine and Hygiene, 104 (2), 519-525. 10.4269 / ajtmh.20-1269.

Fawole, O. I, Okedare, O. O., \& Reed, E. (2021). Home was not a safe haven: women's experiences of intimate partner violence during the COVID-19 lockdown in Nigéria. BMC Women's Health, 21 (32), 1-7. https://doi.org/10.1186/s12905-021-01177-9.

Fineout-Overholt, E., Melnyk, B. M., Stillwell, S. B., \& Williamson, K. (2010). Evidence-Based Practice Step-by-Step: Critical Appraisal of the Evidence: Part I. American Journal of Nursing, 110 (7), 47-52. 10.1097 / 01.NAJ.0000383935.22721.9c.

Gebrewahd, G. T., Gebremeskel, G. G., \& Tadesse, D.B.(2020). Intimate partner violence against reproductive age women during COVID-19 pandemic in northern Ethiopia 2020: a community-based cross-sectional study. Reproductive Health, 17 (1), 1-8. https://doi.org/10.1186/s12978-020-01002-w.

Gibbs, A et al. (2018). Prevalence and factors associated with recent intimate partner violence and relationships between disability and depression in postpartum women in one clinic in eThekwini Municipality, South Africa, Plos One, 12(7). https://doi.org/10.1371/journal.pone.0181236

Gosangi, B., Park, H., Thomas, R., Gujrathi, R., Bay, C. P., \& Raja, A. S. et al. (2021). Exacerbation of Physical Intimate Partner Violence during COVID-19 Pandemic. Radiology, 298(1), 1-8. 10.1148 / radiol.2020202866.

Haq, W., Raza, S. H., \& Mahmood T. (2020). The pandemic paradox: domestic violence and happiness of women. Peer J, 8: e10472. 10.7717 / peerj.10472.

Jahromi, M. K., Jamali, S., Koshkaki, A. R., \& Javadpour, S.(2015). Prevalence and Risk Factors of Domestic Violence Against Women by Their Husbands in Iran. Global Journal of Health Science, 8 (5), 175-183. 10.5539 / gjhs.v8n5p175.

Krishnakumar, A., \& Verma, S. (2021). Understanding Domestic Violence in India during COVID-19: a Routine Activity Approach. Asian Journal of Criminology, 16, 19-35. https://doi.org/10.1007/s11417-020-09340-1.

Krishnamurti, T., Davis, A. L., Quinn, B., Castillo, A. F., Martin, K. L., \& Simhan, H. N. (2021). Mobile Remote Monitoring of Intimate Partner Violence Among Pregnant Patients During the COVID-19 Shelter-In-Place Order: Quality Improvement Pilot Study. Journal of Medical Internet Research. 23 (2). $10.2196 / 22790$.

Mahmood, K. I, Shabu, S. A., M-Amen, K. M., Hussain, S.S., Kako, D. A., \& Hinchliff S., et al. (2021). The Impact of COVID-19 Related Lockdown on the Prevalence of Spousal Violence Against Women in Kurdistan Region of Iraq. Journal of Interpersonal Violence, 1-25. https://doi.org/10.1177/0886260521997929.

Marques, E.S., Moraes, C. L., Hasselmann, M. H., Deslandes, S. F., \& Reichenheim, M. E. (2020). Violence against women, children, and adolescents during the COVID-19 pandemic: overview, contributing factors, and mitigating measures. Caderno de Saúde Pública, 36 (4): 1-6. https://doi.org/10.1590/0102$311 \mathrm{X} 00074420$.

Mascarenhas, M. D. M., Tomaz, G. R., Meneses, G. M. S., Rodrigues, M. T. P., Pereira, V. O. M., \& Corassa, R. B. (2020). Análise das notificações de violência por parceiro íntimo contra mulheres, Brasil, 2011-2017. Revista Brasileira de epidemiologia, 23 (1), 1-13. https://doi.org/10.1590/1980549720200007.supl.1.

Mendonça, M.F.S, \& Ludemir, A. D. (2017). Violência por parceiro íntimo e incidência de transtorno mental comum. Revista de Saúde Pública, 51: 1-8. https://doi.org/10.1590/S1518-8787.2017051006912

Naghizadeh, S., Mirghafourvand, M., \& Mohammadirad, R. (2021). Domestic violence and its relationship with quality of life in pregnant women during the outbreak of COVID-19 disease. BMC Pregnancy Childbirth, 21(88), 1-10. https://doi.org/10.1186/s12884-021-03579-x.

Pattojoshi, A., Sidana, A., Garg, S., Mishra, S. N., Singh, L, K., \& Goyal N., et al. (2021). Staying home is NOT 'staying safe': a rapid 8-day online survey on spousal violence against women during the COVID-19 lockdown in Índia. Psychiatry and Clinical Neurosciences, 75 (2): $63-71.10 .1111$ / pcn.13176.

Paulson, J. L. (2020). Intimate Partner Violence and Perinatal Post-Traumatic Stress and Depression Symptoms: A Systematic Review of Findings in Longitudinal Studies. Trauma, Violence, \& Abuse. 10.1177/1524838020976098. 
Research, Society and Development, v. 10, n. 12, e397101220469, 2021

(CC BY 4.0) | ISSN 2525-3409 | DOI: http://dx.doi.org/10.33448/rsd-v10i12.20469

Roesch, E., Avni, A., \& Gupta J. (2020). Violence against women during pandemic restrictions covid-19. The BMJ, 369. https://doi.org/10.1136/bmj.m1712.

Rosa, D. O. A., Ramos, R. C. S., Gomes, T. M. V., Melo, E. M., \& Melo, V. H. (2018). Violência provocada pelo parceiro íntimo entre usuárias da Atenção Primária à Saúde: prevalência e fatores associados. Saúde Debate, 42 (4), 67-80. https://doi.org/10.1590/0103-11042018S405.

Sabri, B., Hartley, M., Saha, J., Murray, S., Glass, N., \& Campbell, J.C. (2020). Effect of COVID-19 pandemic on women's health and safety: A study of immigrant survivors of intimate partner violence. Health Care for Women International, 41(11-12), 1294- 1312. https://doi.org/10.1080/07399332.2020.1833012.

Santos, C.M., Pimenta, C. A., \& Nobre, M. R. C. (2007). The PICO strategy for the research question construction and evidencesearch. Revista LatinoAmericana de Enfermagem, 15(3), 1-4. https://doi.org/10.1590/S0104-11692007000300023.

Silva, A. F., Estrela, F. M., Soares, C. F. S., Magalhães, J. R. F., Lima, N. S, \& Morais, A. C et al. (2020). Elementos precipitadores/intensificadores da violência conjugal em tempo da Covid-19. Ciências Saúde \& Coletiva, 25 (9), 3475- 3480. https://doi.org/10.1590/1413-81232020259.16132020.

Silva, J. M. M., Lima, M. C., \& Ludermir, A. B. (2017). Intimate partner violence and maternal educational practice. Revista de Saúde Pública, 51, 1-11. https://doi.org/10.1590/S1518-8787.2017051006848.

Souza, M. T. S., Silva, M. D. S., \& Carvalho, R. (2010) Revisão integrativa: o que é e como fazer. Einstein, 8 (1), 102-106. 10.1590/s1679-45082010rw1134.

Vora, M., Malathesh, B. C., Das, S., \& Chatterjee, S. S. (2020). COVID-19 and domestic violence against women. Asian Journal of Psychiatry, 53, 1-2. 10.1016 / j.ajp.2020.102227. 